\title{
The Determination of the Abundances of the Fe Group Elements in Early B Stars from High Resolution FUV Spectra
}

\author{
Geraldine J. Peters ${ }^{1}$, Saul J. Adelman ${ }^{2}$, Ivan Hubeny ${ }^{3}$, Thierry Lanz ${ }^{4}$ \\ ${ }^{1}$ Space Sciences Center \& Dept. of Physics \& Astronomy, \\ University of Southern California, Los Angles, CA 90089-1341, USA \\ email: gjpeters@mucen.usc.edu \\ ${ }^{2}$ The Citadel, Charleston, SC 29409-0270, USA \\ email: adelmans@citadel.edu \\ ${ }^{3}$ University of Arizona, Tucson, AZ 85721-0065, USA \\ email: hubeny@aegis.as.arizona.edu \\ ${ }^{4}$ University of Maryland, College Park, MD 20742-2421, USA, \\ email: lanz@astro.umd.edu
}

\begin{abstract}
We present selected results from an investigation that is currently underway to determine the abundances of the Fe group elements in early B stars and assess the extent to which contemporary NLTE and LTE models represent their atmospheres. High resolution UV and optical spectra of B stars that display ultrasharp lines are compared with computations from TLUSTY/SYNSPEC and SYNTHE. Some results from our analysis of the abundance standard $\iota$ Her $(\mathrm{B} 3 \mathrm{~V})$ are presented here.
\end{abstract}

Keywords. stars: abundances, stars: atmospheres, stars: early-type

\section{Overview of Project}

Historically the atmospheres of B-type main-sequence band stars have provided perhaps the most accurate information on the present-day abundances of the elements in a galaxy and its chemical evolution. We are currently determining the abundances of light and Fe group elements in a subset of early B stars that display exceptionally sharp lines and investigating the extent to which contemporary NLTE and LTE models represent their atmospheres. The abundances of the Fe group elements are of particular interest, as they serve as a tracer of earlier supernova activity across a galaxy. High resolution spectral data from HST, FUSE, and the KPNO Coudé Feed Telescope are compared with computations from the NLTE codes TLUSTY/SYNSPEC (Hubeny 1988, Hubeny \& Lanz 1995) and the LTE programs ATLAS9/SYNTHE (Kurucz 1993ab). The atmospheric parameters $\mathrm{T}_{\text {eff }}$, $\log g$ are determined in standard ways from the SED, Balmer line profiles, and the ionization balance criterion, while the microturbulence, $\xi_{\text {turb }}$, is assessed by the method of examining the abundances versus the EWs of the lines of $\mathrm{O}$ II and other ions or from the SED. Some results for $\iota$ Her (B3V) are presented below.

\section{2. $\iota$ Herculis}

$\iota$ Her is an ultrasharp-lined B3V field star that has long been considered an abundance standard. To determine the abundances of the Fe group elements, we obtained FUV/NUV spectra of $\iota$ Her with the $H S T /$ STIS E140M/E230M echelle gratings as part of a $H S T$ SNAPSHOT program. Other than for a few weak multiplets of Fe III in the optical region, 

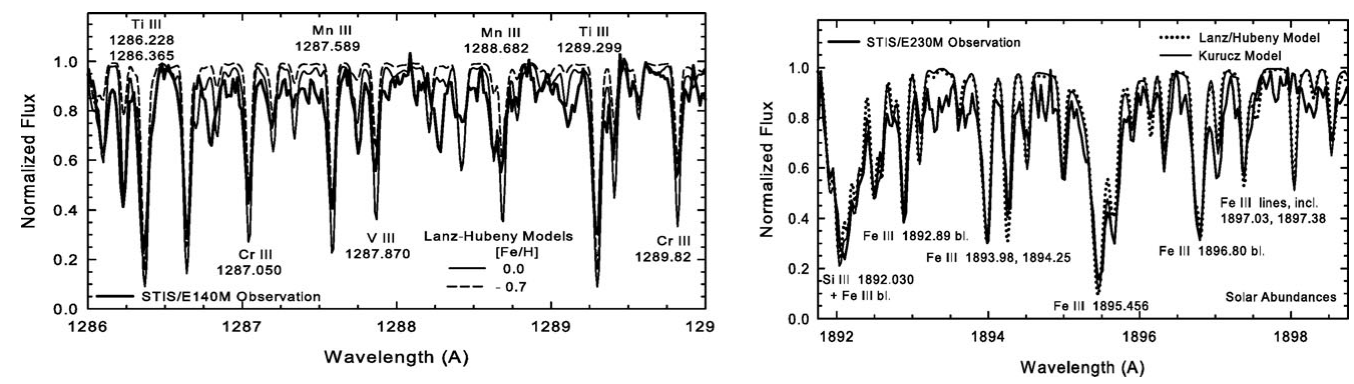

Figure 1. The observed UV spectrum of $\iota$ Her compared with model spectra computed from TLUSTY/SYNSPEC and ATLAS9/SYNTHE. Left panel: Fits in a region that contains numerous lines of Ti III, Cr III, and Mn III suggest abundances that are $\sim 0.3-0.7$ dex below solar values. Right panel: A solar Fe abundance is implied by fits to Fe III lines in the $1900 \AA$ region. There is good agreement between NLTE \& LTE calculations. Model parameters are $T_{\text {eff }}=17500 \mathrm{~K}$, $\log g=3.75, \xi_{\text {turb }}=0 \mathrm{~km} \mathrm{~s}^{-1}$, and $\mathrm{V} \sin i=0 \mathrm{~km} \mathrm{~s}^{-1}$.

the measurable lines from the Fe group are typically found in the UV. Model spectra were computed with the Hubeny/Lanz NLTE and the Kurucz LTE codes and compared with the continuum-normalized observations. In Fig. 1 we show sample spectral fits in the 1286-1290 $\AA$ and 1892-1899 $\AA$ regions that are rich in lines from the Fe group. The grid of B star models by Lanz \& Hubeny (2007) were used for the NLTE calculations. Fe was treated in NLTE. The calculations for the other Fe group elements were made in the LTE approximation. Most fits to the lines of Ti III, Cr III, and Mn III (cf. Fig. 1) suggest abundances that are in the range of 0.3-0.7 dex below the solar values given in Asplund et al. (2005). The numerous Fe III lines in the 1880-2000 $\AA$ region were fit well with a solar abundance (Fig. 1), and there was good agreement between the NLTE and LTE calculations. But other lines of this species (e.g. near $1400 \AA$ ) were computed to be too strong for both NLTE and LTE treatments. Besides its sharp lines, $\iota$ Her makes an excellent abundance standard because its microturbulence appears to be near zero. Typically UV lines in early B stars suggest a smaller value of $\xi_{\text {turb }}$ than the optical O II features, and this causes most of the prominent UV lines to suggest lower abundances, but this is not a problem here. However it should be mentioned that the lines in $\iota$ Her are much sharper than the spectral resolution of the E140M and E230M gratings $\left(\sim 7-10 \mathrm{~km} \mathrm{~s}^{-1}\right)$ and observations with the E140H and E230H echelle gratings, which have a resolution of $\sim 3 \mathrm{~km} \mathrm{~s}^{-1}$, are warranted to investigate photospheric velocity fields and improve the abundance determinations.

The authors appreciate support from NASA grants NAG5-11802, NAG5-12239, NAG513212, and STScI grants GO-09848 \& GO-06709.

\section{References}

Asplund, M., Grevesse, N., \& Sauval, A. J. 2005, in F. N. Bash \& T. G. Barnes (eds.), Cosmic Abundances as Records of Stellar Evolution and Nucleosynthesis (ASP Conf. Ser. 336,San Francisco: ASP), p. 25

Hubeny, I. 1988,Comput. Phys. Commun. 52, 103

Hubeny I \& Lanz, T. 1995 ApJ, 439, 875

Kurucz, R. L. 1993a,Kurucz CD-ROM No. 13, (Cambridge:SAO)

Kurucz, R. L. 1993b,Kurucz CD-ROM No. 18, (Cambridge:SAO)

Lanz T. \& Hubeny, I. 2007 ApJS, 169, 83 